\title{
CORRIGENDUM
}

\section{Enhanced hypertension prevalence in non-Han Chinese minorities from Xinjiang Province, China}

Zhensui Lu, Zhenhong Lu, Yanling Zhu, Zhaoyang Yan, Xiaoquan Liu, Shigui Yan and Guo-Ping Shi

Hypertension Research (2010) 33, 90; doi:10.1038/hr.2009.204

Correction to: Hypertension Research (2009) 32, 1097-1103;
doi:10.1038/hr.2009.159

The authors of the above article noted an error in publication of this paper (AOP and in the December 2009 issue) in the list of authors and the corresponding authors. The correct list of authors and corresponding authors is shown below.

Zhensui $\mathrm{Lu}^{1,2}$, Zhenhong $\mathrm{Lu}^{3}$, Yanling $\mathrm{Zhu}^{4}$, Zhaoyang $\mathrm{Yan}^{5}$, Xiaoquan Liu ${ }^{4}$, Weiqi Yan $^{1,2}$ and Guo-Ping Shi ${ }^{6}$
Correspondence: Dr G-P Shi, Cardiovascular Medicine, Brigham and Women's Hospital, Harvard Medical School, NRB-7, 77 Avenue Louis Pasteur, Boston, MA 02115, USA.

E-mail: gshi@rics.bwh.harvard.edu or Dr W Yan, Clinical Research Center, The Second Affiliated Hospital, Medical College, Zhejiang University, Hangzhou 310009, China.

E-mail: wyan@zju.edu.cn 